\title{
A fast SPIHT algorithm based on the end scan threshold
}

\author{
Liu Changhua $^{1, \text { a }}$, Xu Xiaole ${ }^{1, b}$ \\ ${ }^{1}$ Mathematics and Computer institution, Wuhan Polytechnic University, Wuhan, China \\ aliuch@whpu.edu.cn, ${ }^{\text {b } 598962960 @ q q . c o m ~}$
}

Keywords: Image compression, SPIHT, End scan threshold, minimum output bits

\begin{abstract}
A fast set partitioning in hierarchical tree (SPIHT) algorithm based on the end scan threshold is proposed in this paper. Because the coding process of the SPIHT needs repeat scanning and sorting, so it takes a lot of extra time and storage space. In order to save the time and space overhead, the end scan threshold is used in encoding to terminate the scan process when the compression is enough. Experimental results show that the end scan threshold for the different images is the same in the case of the same compression ratio, and when the improved algorithm is used in the sorting process, it can reduce the data stream storage space in coding and the time consumption and decoding. In addition, the quality of reconstructed image is equivalent to SPIHT algorithm.
\end{abstract}

\section{Introduction}

With the development of satellite remote sensing imaging and telecommunication technologies, a large number of image information produced, thus the image compression technology has become an important issue. Due to the characteristics of the wavelet transform, it can be applied in the field of image compression [1-3]. Shapiro $M$ Jeromein introduced wavelet transform in image compression and put forward embedded zero tree wavelet (EZW)coding[4,5]. In 1996 Said A and Pearlman WA proposed SPIHT algorithm[6] based on Shapiro's EZW algorithm to solve the shortcomings of EZW algorithm, but the repeated scanning in the SPIHT algorithm requires a large amount of data storage space and long coding time. When faced with a complex texture image, its problem of rescanning in sorting will largely influence the real-time of image compression. At the same time, the problem in data storage is also highlighted because of rescanning. So it is not suitable for the real-time compression of complex texture image.

In this article, we again explain that the SPIHT technique[7-9]. It's crucial parts are coding process - the way subsets of coefficients are partitioned and how the significance information is conveyed. We say that a coefficient is significance or insignificance with respect to a given threshold, depending on whether or not it exceeds the threshold. Here, the subset partitioning is so effective and the significance information so compact that even binary uncoded transmission achieves about the better performance than the SPIHT. On the other hand, a large fraction of the "bit-budget" is spent in the sorting pass, and it is there that the sophisticated coding methods are needed.

In this work, we propose the concepts of end scan threshold and minimum output bits. They can be used to solve the problem of SPIHT algorithm repeatedly scanning and large comsumption in storage capacity, saving coding time and storage space, so it can be well applied to the real-time compression of image.

This paper is organized as follows: section II gives the algorithm description, all the steps of the improved algorithm are described in section II where we introduce the end scan threshold, and it presents how the data streams can be produced using the proposed method; experimental results and discussion are presented in section III, and we will display the reconstruct images, the value of the PSNR and the coding time are also caculated; and finally we will concludes the paper in section IV. 


\section{Algorithm Description}

\section{The concept of the end scan threshold}

In the coding of the SPIHT algorithm, the initial scan threshold is the determinded value based on the calculation of wavelet coefficients, and it will halved on the basis of the previous threshold successively. Because of the characteristics of the wavelet transform, the energy information of image exists in the low-frequency part of the wavelet decomposition[10-13].

With the cycle scan going, the scan threshold is getting smaller and the former unimportant coefficients will become important. On the other hand, each scan will output the significant bit of the important coefficients. When the compression ratio is constant, the scanning threshold is reduced to a certain value, it terminates the scanning and stops encoding.

Therefore, we take four images for studying the imortant coefficients when the threshold is different. In this progress, the important coefficients will be counted at different threshold. The four image are 512*512 Lena, Goldhill, Barbara and 256*256 Cameraman. The data will be shown in the Table 1.

Table 1 SPIHT for image important coefficients statistics

\begin{tabular}{|c|c|c|c|c|}
\hline $\mathrm{T}$ & Lena & Goldhill & Barbara & Cameraman \\
\hline 128 & 2013 & 1994 & 1554 & 570 \\
\hline 64 & 3545 & 5510 & 3517 & 913 \\
\hline 32 & 4932 & 14642 & 6497 & 1934 \\
\hline 16 & 15178 & 32598 & 16888 & 3891 \\
\hline 8 & 33650 & 61622 & 40943 & 7973 \\
\hline 4 & 48856 & 78625 & 71268 & 13302 \\
\hline 2 & 77589 & 106325 & 108131 & 19295 \\
\hline 1 & 133093 & 183628 & 154059 & 28400 \\
\hline
\end{tabular}

Table 1 shows that the important coefficients are increasing as the threshold value decreasing. In the actual scan, it's not necessary for the scan cycle threshold reduced to 1 and then stops scanning. That will produce a lot of scan data, and these data don't have too much practical significance to image compression. In contrast, repeatedly scanning requires will extend scanning time and take more storage space. That's why the concept of end scan threshold was introduced in this paper. In the situation of setting the compression ratio, the end scan threshold is an identified value, when the scanning is reduced to the end scan threshold value, cyclic scanning can be stopped.

\section{The end scan threshold setting}

Introducing end scan threshold has great help for reducing coding time and saving data storage capacity. Meanwhile we define minimum output bits based on the end scan threshold. It shows in equation (1).

$$
b i t_{\min }=\log _{2} T_{\mathrm{e}}
$$

In formula (1), $T_{\mathrm{e}}$ is the end scan threshold.

After a number of experiments, Table 2 shows the end scan threshold of these images under different compression ratio.

The results shows that the end scan threshold has the following characteristics:

(1)Different image has the same end scan threshold under the same compression ratio

(2)The end scan threshole is growing as the data of compression ratio is increasing.

(3)The end scan threshole for same image is different under different compression ratio.

In the process of SPIHT coding, we set different scan threshold for determining the important coefficients. The end scan threshold is certain when the $\mathrm{Cr}$ is given, so the minimum output bit can be calculated. This step is very important because the distance between the most significant bit and the minimum output bit will be calculated in refinement scanning. And then we can output the data flow bit before the minimum output bit, so the LSP list is saved. After this step processing, the original three data list is reduced to two. This can greatly reduce the number of scan times and save the data storage space. Meanwhile it can greatly reduce encoding time. 
Table 2 The end scan threshold at different compression ratio

\begin{tabular}{|c|c|c|c|c|c|c|c|c|}
\hline \multirow{2}{*}{ Cr } & \multicolumn{2}{|c|}{ Lena } & \multicolumn{2}{c|}{ Goldhill } & \multicolumn{2}{c|}{ Barbara } & \multicolumn{2}{c|}{ Cameraman } \\
\cline { 2 - 9 } & 512 & 256 & 512 & 256 & 512 & 256 & 512 & 256 \\
\hline 2 & 1 & 1 & 1 & 1 & 1 & 1 & 1 & 1 \\
\hline 4 & 4 & 4 & 4 & 4 & 4 & 4 & 4 & 4 \\
\hline 6 & 8 & 8 & 8 & 8 & 8 & 8 & 8 & 8 \\
\hline 8 & 16 & 16 & 16 & 16 & 16 & 16 & 16 & 16 \\
\hline 10 & 32 & 32 & 32 & 32 & 32 & 32 & 32 & 32 \\
\hline
\end{tabular}

For example, if the maximum wavelet coefficients is between 128 and 256, the initial threshold can be calculated during the initialization process. It is 128 and the threshold index is 7 . At the same time ,if the end scan threshold is 4 , the minimum output bit is 2 . So all of the significant coefficients have been determined which can be directly outputted the first $(7+1-2)=6$ data bits flow.

\section{Improved SPIHT coding algorithm flowchart}

Fig. 1 shows that the improved SPIHT coding algorithm flowchart. We can know each step in the improved algorithm. The detailed description of the improved algorithm is following in the next section.

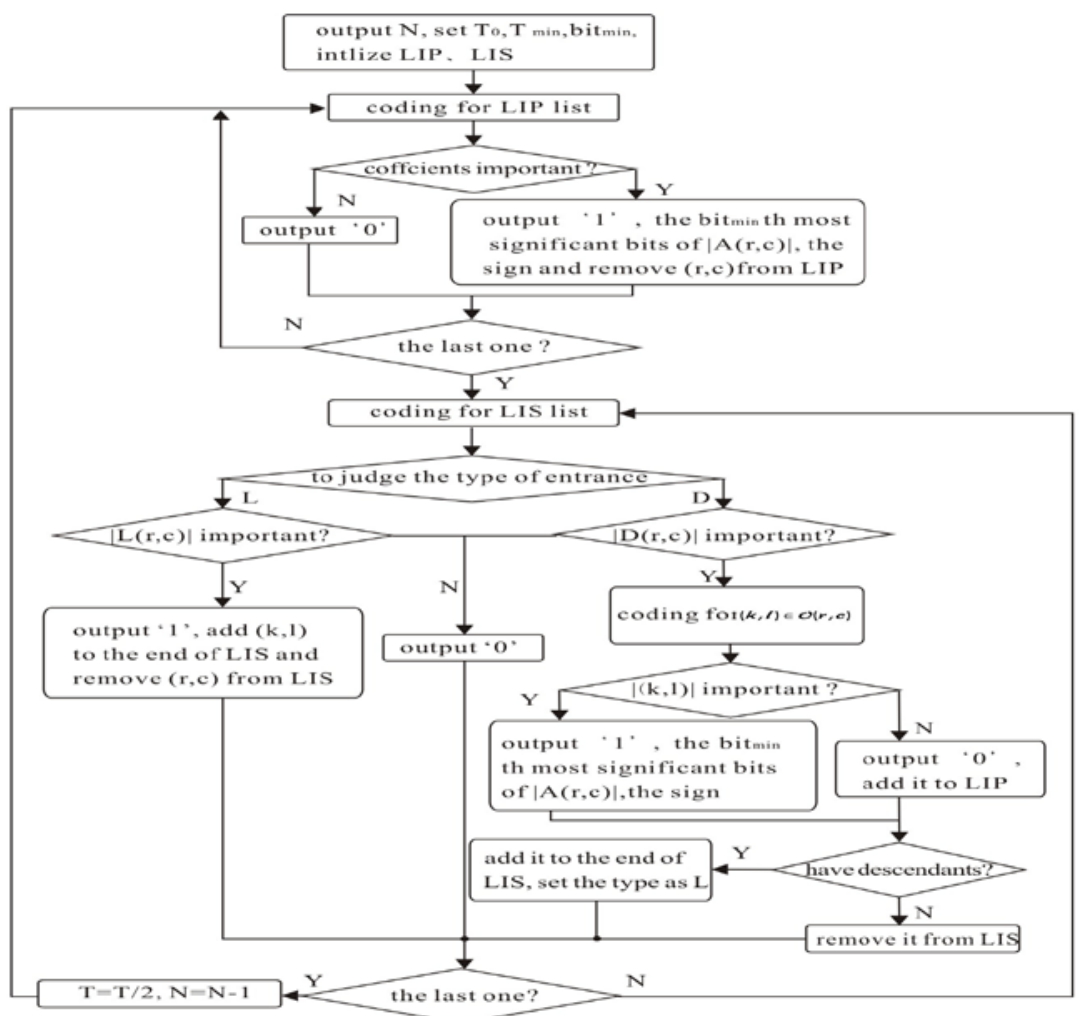

Fig. 1 The Improved SPIHT coding algorithm flowchart

\section{Algorithm Description}

After $\mathrm{N}$-level wavelet transform for the image, we can get $3 \mathrm{~N}+1$ subbands and a"spatial orientation tree [5-7]" structure is formed with sorting the band according to the order from low frequency to high frequency. Root is the most low-frequency Sub-band node. Each node of the tree corresponds to a wavelet coefficient. It shows in equation (2).

$$
\left\{\begin{array}{l}
Z(r, c)=(r, c)+D(r, c) \\
D(r, c)=O(r, c)+L(r, c) \\
L(r, c)=\sum D(k, l) \quad(k, l) \in O(r, c)
\end{array}\right.
$$

In equation (2), $(r, c)$ are the coordinates of the Wavelet coefficients, $A(r, c)$ are the node or 
coefficients, $O(r, c)$ are the coordinates of all offspring of node $(r, c), D(r, c)$ are the coordinates of all the descendants of the node $(r, c)$.

We define I as the set of coordinates of all spatial orientation tree roots (nodes in the highest pyramid level). So we can know that the $(r, c) \in I$.

In the scanning progress of the improved algorithm, we use the same set partitioning rules as is used in the SPIHT. The following paragraphs will give us a brief description about the rules.

(1) The initial coordinate sets are the $\{(r, c)\}$ and $D(r, c)$, for all $(r, c) \in I$.

(2) In the current threshold, if $D(r, c)$ is important, so it is partitioned into $L(r, c)$ and $L(r, c)$ is split into the four single-element sets with $(k, l) \in O(r, c)$.

(3) If $L(r, c)$ is important under the current scaning threshold, then it is split into the four sets $D(k, l)$, with $(k, l) \in O(r, c)$.

$\mathrm{X}$ is defined as a Wavelet coefficients set: $X=\{|A(r, c)|\}$. To make clear the relationship between magnitude comparisons and message bits, we use the function:

$$
G_{n}(X)=\left\{\begin{array}{l}
1, \max \{|A(r, c)|\} \geq T \\
0, \quad \text { otherwise }
\end{array}\right.
$$

In the function, if $G_{n}(X)=1$, that means the set of $X$ is important when the threshold is T. To simplify the notation of single pixe sets, we write $G_{n}(\{(r, c)\})$ as $G_{n}(r, c)$.

Because the set will be tested whether it matters according to the order, so the improved algorithm will give two ordered lists for storing the significance information. They are called list of insignificant sets (LIS), list of insignificant pixels (LIP). In the two lists, each entry is identified by a coordinate $(r, c)$, which in the LIP represents individual pixels, and in the LIS represents either the set $D(r, c)$ or $L(r, c)$. To make a difference between them, we define that a LIS entry is of type ' $D$ ' if it represents $D(r, c)$, and of type ' $L$ ' if it represents $L(r, c)$.

Improved SPIHT algorithm encoding process:

(1)Initialization progress:

output $\mathrm{N}=N=$ floor $\left(\log _{2}^{\max \{|A(r, c)|\}}\right)[14]$; set the value of $T_{0}, T_{\mathrm{e}}$, bit $t_{\text {min }}$, and initialize the LIP, LIS.

(2)Scanning coding:

2.1)LIP chain coding. for each entry $(r, c)$ in the LIP do:

2.1.1)output $G_{n}(r, c)$;

2.1.2)if $G_{n}(r, c)=1$ then add ' 1 ' and the sign of $A(r, c)$ to $S n$, then output the Significant bits of binary bits of important coefficients before bit $t_{\min }$ to $\mathrm{Rn}$ and move $(r, c)$ out of the LIP; if $G_{n}(r, c)=0$ then add ' 0 ' to $\mathrm{Sn}$;

2.2)LIS chain coding. For each $(r, c)$ in the LIS do:

2.2.1)if the entity $(r, c)$ is of type ' $D$ ' then output the $G_{n}(D(r, c))$

if $G_{n}(D(r, c))=1$ then add ' 1 ' to Sn for each $(k, l) \in O(r, c)$ do: output $G_{n}(k, l)$;

if $G_{n}(k, l)=1$ then output the Significant bits of binary bits of important coefficients before bit $_{\min }$ to Rn and move $(k, l)$ out of the LIS;

if $G_{n}(k, l)=0$ then add $(k, l)$ to the end of LIP;

if $L(r, c) \neq \varnothing$ then move $(r, c)$ to the end of the LIS, as an entry of type ' $L$ ', and go to the step2.2.2; otherwise, remove entry $(r, c)$ from the LIS;

2.2.2)if the entry is of type ' $L$ ' then

output $G_{n}(L(r, c))$; if $G_{n}(L(r, c))=1$ then

add each $\left(r_{o}, c_{o}\right) \in O(r, c)$ to the end of the LIS as an entry of type ' $\mathrm{D}$ ', remove $(r, c)$ from the 
LIS.

$$
\text { if } G_{n}(L(r, c))=0 \text { then add ' } 0 \text { ' to Sn. }
$$

(3)Quantization-step update:decrement $\mathrm{N}$ by 1 and go to step 2 until the threshold to $T_{\mathrm{e}}$, then stop the coding.

\section{Experimental results and analysis}

The end scan threshold will be set based on the data of Table 2 when the compress ratio is 2,4,6,8,10.We use 512*512 Barbara and Lena ,256*256 Cameraman for coding experiment. Comparing the PSNR and coding time with SPIHT algorithm and JPEG2000 algorithm when the $\mathrm{Cr}$ is the same. Experimental results have shown at the Fig2,table3 and table4. The D5/3 wavelet basis is adopted into the wavelet decomposition.

We will compare the recovered image with the original, and also compare the reconstruct image for different coding scheme when the compress ratio is 2. Then the PSNR is calculated, because the distortion is measured by the peak signal to noise ratio.

$$
P S N R=10 \log _{10}\left(\frac{255^{2}}{M S E}\right) d B
$$

Where MSE denotes the mean squared-error between the original and reconstructed images. Finally, the commparsion of execution time for different coding scheme is counted.

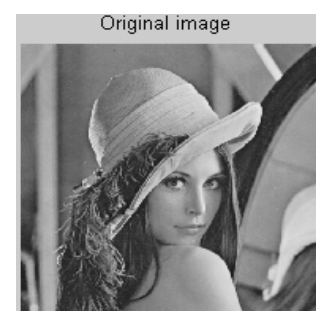

(a)

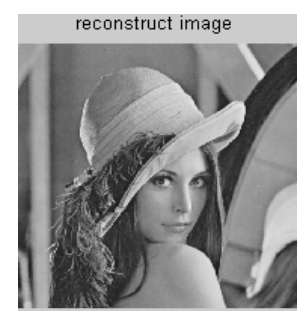

(b)

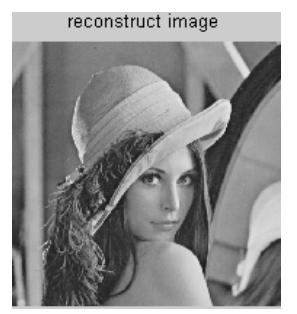

(c)

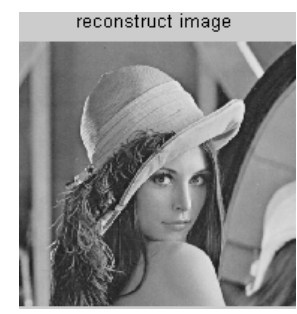

(d)

Fig 2 Comparison of the reconstruct image for different coding schem

(a)Original image Lena(b)SPIHT reconstruct image(c)JPEG2000 reconstruct image(d)Improve scheme reconstruct image

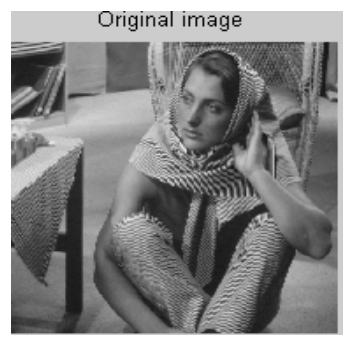

(a)

\section{(b)}

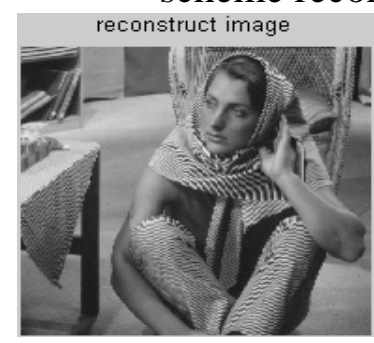

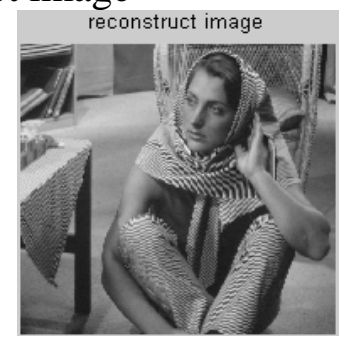

(d) (c)

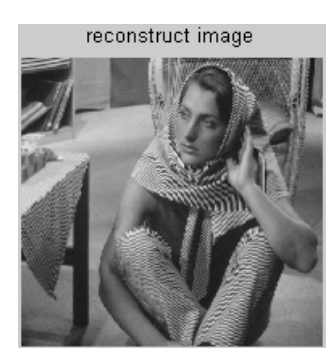

ding schem

(a)Original image Barbara (b)SPIHT reconstruct image (c)JPEG2000 reconstruct image (d) Improve scheme reconstruct image 
Table 3 comparison of psnr performances for different coding schemes (dB)

\begin{tabular}{|c|c|c|c|c|c|c|c|c|c|}
\hline \multirow{2}{*}{ Cr } & \multicolumn{3}{|c|}{ Barbara } & \multicolumn{3}{c|}{ Lena } & \multicolumn{3}{c|}{ Cameraman } \\
\cline { 2 - 10 } & $\mathrm{a}$ & $\mathrm{b}$ & $\mathrm{c}$ & $\mathrm{a}$ & $\mathrm{b}$ & $\mathrm{c}$ & $\mathrm{a}$ & $\mathrm{b}$ & $\mathrm{c}$ \\
\hline 2 & 37.35 & 38.23 & 39.38 & 39.95 & 41.12 & 42.86 & 40.62 & 41.33 & 43.03 \\
\hline 4 & 34.26 & 35.12 & 36.21 & 38.76 & 39.66 & 41.03 & 39.14 & 40.06 & 41.07 \\
\hline 6 & 32.03 & 33.35 & 34.48 & 36.55 & 37.14 & 39.62 & 37.26 & 38.15 & 39.53 \\
\hline 8 & 27.83 & 28.62 & 29.76 & 34.88 & 35.16 & 37.14 & 34.79 & 35.01 & 36.86 \\
\hline 10 & 26.46 & 27.33 & 28.35 & 31.96 & 31.05 & 34.51 & 33.13 & 32.98 & 34.08 \\
\hline
\end{tabular}

Table 4 comparison of execution time for different coding schemes(time:s)

\begin{tabular}{|c|c|c|c|c|c|c|c|c|c|}
\hline \multirow{2}{*}{ Cr } & \multicolumn{3}{|c|}{ Barbara } & \multicolumn{3}{c|}{ Lena } & \multicolumn{3}{c|}{ Cameraman } \\
\cline { 2 - 10 } & $\mathrm{a}$ & $\mathrm{b}$ & $\mathrm{c}$ & $\mathrm{a}$ & $\mathrm{b}$ & $\mathrm{c}$ & $\mathrm{a}$ & $\mathrm{b}$ & $\mathrm{c}$ \\
\hline 2 & 4.78 & 3.62 & 2.31 & 4.44 & 3.13 & 1.39 & 3.91 & 2.86 & 1.31 \\
\hline 4 & 3.01 & 2.31 & 1.58 & 3.04 & 2.14 & 1.05 & 3.10 & 1.95 & 0.96 \\
\hline 6 & 2.40 & 1.98 & 1.32 & 2.06 & 1.76 & 0.97 & 2.12 & 1.63 & 0.54 \\
\hline 8 & 1.96 & 1.86 & 0.95 & 1.73 & 1.68 & 0.75 & 1.05 & 0.99 & 0.26 \\
\hline 10 & 0.98 & 0.95 & 0.47 & 0.85 & 0.83 & 0.34 & 0.64 & 0.59 & 0.13 \\
\hline
\end{tabular}

In Fig2 and Fig3, they show that the original image, reconstruct image based on SPIHTand reconstruct image based on improved scheme. There are two images for testing.

As can be seen from the Table 2, texture details of the Barbara image are plentiful, while Lena is the general image which has relatively simple texture. With introducing end scan threshold, the PSNR of the SPIHT algorithm and JPEG2000 are almost same, the JPEG2000 is the better one .The PSNR of the improve algorithm is $1 \mathrm{~dB}$ bigger compared with the SPIHT and JPEG2000 at average. In other words, The image restoration quality of improve algorithm is the best. And the coding time is obviously reduced. The coding time of Barbara image, Lena image and Camera image respectively is about $1 / 2,1 / 3,1 / 4$ of the SPIHT algorithm. Comparing with the JPEG2000, the improve algorithm also has the advantage.

\section{Conclusion}

In this paper, we present the conceptions of the end sacn threshold and the minimum output bits. Then we give an improving scheme. This scheme will set the end scan threshold and calculate the minimum output bits at the beginning of the sorting pass. In detail, by borrowing the improving algorithm, we can not only achieve the initial threshold but also determine when the scaning can be finished. The analysis results show that the end scan threshold of different images are approximately equal under the same compression ratio .

After introducing end scan threshold, we do not need the LSP list and so the storage space reduced greatly. Meanwhile, under the condition of the same compression ratio, we know the end scan threshold so that we known when to stop the coding. This can save the coding time greatly. as well as the coding and decoding time with the same compression performance, it is beneficial to the real-time image compression.

With the development of high-resolution satellite imaging technology, the amount of data of high- resolution satellite images increase quickly. Also the most of these images texture details is abundant .If using the general compression algorithm to deal with it,it takes long time and large storage space to process scan sort. When the image texture details is rich, improved algorithm can 
save compression time ,making the advantages of satellite images compression for high resolution texture details obvious and having enough research space. On the other hand, the improved algorithm can be used in reference picture compression of stereo pairs in the future. Making the improved algorithm can be combined with compression algorithms of target figure. Improving its compression performance. It will be key consideration in subsequent study.

\section{Acknowledgments}

This work was supported by the National Science Foundation for Young Scientists of China(No.61201452); the Graduate Innovation Fund of Wuhan Polytechnic University(Grant No. 2013cx007).

\section{References}

[1] Chen Wufan, Yang Feng, Jang Guiping, Wavelet Analysis and Its Application on Image Processing, Beijing:Publishing House of Science, 2002.

[2] Rafael C Gonzalez, Richard E Woods. Digital image processing.2nd , Beijing:Publishing House of Electronics Industry,2002.

[3] Antonini M,Barlaud M,Mathieu P,et al, Image coding using wavelet transform, IEEE Trans Image Processing,vol. 1, pp.719-746,1992.

[4] Shapiro J M, Embedded image coding using zerotree of wavelets coefficients, IEEE Trans Signal Processing,vol. 41, pp.3445-3462, 1993.

[5] Xia yan-hui, Ma wen-bo, An Improved Fast Algorithm of EZW Based on Wavelet Transform,Journal of LASER \& INFRARED, vol. 39, pp.110-112, 2009.

[6] Amir Said,William A Pearlman, A new,fast,and efficient image code based on set partitioning in hierarchical trees, IEEE Transactions on Circuits and Systems for Video Technology, vol. 6, pp.2434-250, 1996.

[7] M. Santhi,R. S.D. Wahida Banu,M. Santhi, Enhancing the Color Set Partitioning in Hierarchical Tree (SPIHT) Algorithm Using Correlation Theory, Journal of Computer Science,vol. 7, pp.1245-1250, 2013.

[8] Brahimi, T,Melit, A,Khelifi, F et al, An improved SPIHT algorithm for lossless image coding, Digital Signal Processing, vol. 19, pp.220-228, 2009.

[9] Deng Chen-wei, Zhao Bao-jun, An Approach to Modify Fast SPIHT Algorithm, Transactions of Beijing Institute of Technology, vol. 30,pp.478-482, 2010.

[10] Jia zhi-ke, Cui hui-juan, Tang kun, Improved SPIHT still image compression algorithm, Journal of Tsinghua University, vol. 41, pp.25-28, 2001.

[11] Wang yong-yu, Sun qu, Yuan chao-wei, Power system fault recording data compression based on IWT and SPIHT coding, Journal of Relay, vol. 36, pp.50-54, 2008.

[12] Qin Xiangpei, Zhen Xianzhong. Image processing Collection Based on MATLAB, Beijing: Publishing House of Science, 2011.

[13] Xiaohong Zhang, Yuanyuan Zhu, Zhiyong Cui, Image Compression Quantification Scheme Based on Lifting Wavelet Technology, Journal of System Simulation, vol. 23, pp.2434-2438, 2011. 